13

\title{
Квантовая теория эмиссии электронов из структуры „металл-диэлектрик“ в сильных электрических полях
}

\author{
() С.И. Берил, ${ }^{1}$ С.А. Баренгольц, ${ }^{2,3,9}$ Ю.А. Баренгольц, ${ }^{1}$ А.С. Старчук ${ }^{1}$ \\ ${ }^{1}$ Приднестровский государственный университет им. Т.Г. Шевченко, \\ 3300 Тирасполь, Молдова, Приднестровье \\ ${ }^{2}$ Институт общей фризики им. А.М. Прохорова РАН, \\ 119991 Москва, Россия \\ ${ }^{3}$ Физический институт им. П.Н Лебедева РАН, \\ 119991 Москва, Россия \\ ฯ e-mail: sabarengolts@mail.ru
}

Поступило в Редакцию 25 декабря 2018г. В окончательной редакции 4 ноября 2019г. Принята к публикации 13 января 2020г.

\begin{abstract}
Выведена обобщенная формула для тока электронной эмиссии как функции температуры, поля и работы выхода электрона в системе „металл-диэлектрик“ с учетом квантового характера сил изображения. Для свободных электронов использовано распределение Ферми-Дирака и квантовый потенциал изображения, полученный методами электронной поляронной теории. В пределе классического потенциала сил изображения получены хорошо известные формулы Ричардсона-Шоттки - для термоэлектронной эмиссии и Фаулера-Нордгейма - для автоэлектронной эмиссии. Показано, что при высоких температурах и электрических полях $E \geq 10 \mathrm{MV} / \mathrm{cm}$ поляронный вклад растет с ростом поля и снижается с ростом температуры. Уменьшение эмиссионного тока связано с увеличением эффективной работы выхода электрона, обусловленным электронным поляронным эффектом. Для тока термо- и автоэлектронной эмиссии получены удобные для теоретических оценок экстраполяционные формулы.
\end{abstract}

Ключевые слова: термоэлектронная эмиссия, автоэлектронная эмиссии, электронный полярон, квантовый потенциал изображения.

DOI: 10.21883/JTF.2020.06.49295.441-18

\section{Введение}

Исследование вакуумного пробоя показало, что важную роль в инициировании вакуумного разряда играют эмиссионные процессы, стимулированные неметаллическими включениями (островковые или сплошные диэлектрические пленки, адсорбированные атомы и т.д.) на поверхности металлического катода [1]. Именно с наличием таких включений связывают существование аномально высоких коэффициентов усиления электрического поля $\beta$, определяемых из характеристик Фаулера-Нордгейма [2]. Значения этих коэффициентов могут достигать нескольких сотен [3]. Эмиссионные процессы с участием этих неметаллических включений приводят к образованию так называемых катодных пятен 1-го типа: короткоживущих источников плазмы, генерируемой в межэлектродный промежуток [4,5]. Функционирование пятен 1-го типа во многих случаях определяет начальную стадию вакуумного пробоя.

Вопрос корректного описания процесса эмиссии с катода, покрытого неметаллическими включениями, и их роли в инициировании вакуумного пробоя в последнее время приобрел важное значение в связи с разработкой электрон-позитронных коллайдеров $\mathrm{TeV}$-диапазона энергий [6]. Инициирование вакуумного пробоя на по- верхности ускоряющей структуры является основной проблемой, ограничивающей темп набора энергии частицами [7]. Возможно, что именно присутствие неметаллических включений на поверхности ускоряющей структуры ответственно за существование аномально высоких коэффициентов электрического поля. Эмиссионные центры с высокими $\beta$ являются потенциальными источниками взрывной электронной эмиссии, которая, по-видимому, и является основной причиной вакуумного пробоя в ускоряющих структурах [8].

Задача корректного описания эмиссионных процессов с участием неметаллических включений актуальна для изучения начальной стадии развития высоковольтного разряда в газовых средах [9]. В этом случае, учитывая наличие газовой среды, такие включения неизбежно присутствуют на поверхности катодов.

В настоящей работе эмиссионные процессы в структуре металлический катод-адсорбированная неметаллическая пленка-вакуум (газовая среда) рассмотрены на основе квантово-механического описания взаимодействия электрона с быстрой поляризацией среды (колебания плазмы валентных электронов диэлектрика и свободных электронов металла). При этом туннелирующий электрон представляет собой квазичастицу - электронный полярон Тоезавы, имеющий конечные размеры, опре- 
деленные радиусом $R_{p}$ [10-12]. Теория поверхностного электронного полярона, на основе которой рассчитаны его параметры, приведена в работе [13].

Этот подход позволил получить выражение для потенциальной энергии взаимодействия электрона с наведенной им поляризацией среды при исследовании эмиссии электронов из металла в диэлектрик (газовую среду) [14-17], справедливое во всем диапазоне значений расстояния $x$ электрона до границы раздела металла с диэлектриком, в том числе в точке $x=0$ (граница раздела), в которой классический потенциал сил изображения расходится. В пределе $x \gg R_{p}$ этот квантовый потенциал переходит в электронную часть классического потенциала сил изображения $U_{i e}(x)=-e^{2} /(4 \varepsilon x)$, где $\varepsilon-$ высокочастотная диэлектрическая проницаемость диэлектрика.

Структура настоящей работы следующая: в первой части приведен вывод обобщенной формулы для эмиссионного тока через контакт металл-диэлектрик с учетом электронного поляронного эффекта в зависимости от приложенного электрического поля и температуры катода. Далее рассмотрены предельные случаи высоких температур (термоэлектронная эмиссия) и электрических полей (автоэлектронная эмиссия). Определены параметры эмиссии электронов и диэлектрических пленок, при которых вклад электронного поляронного эффекта становится существенным. Получены экстраполяционные формулы для случаев термоэлектронной и автоэлектронной эмиссии, переходящие в пределе классического потенциала изображения в известные формулы Ричардсона-Шоттки и Фаулера-Нордгейма соответственно.

\section{Основные уравнения}

Плотность эмиссионного тока из структуры металл-диэлектрик (рис. 1, $a$ ) можно выразить формулой [18]

$$
j(E, T)=e \int_{-\infty}^{\infty} N(W, T) D(W, T) d W .
$$

Здесь $j(E, T)$ - плотность эмиссионного тока, $e-$ заряд электрона, $E-$ напряженность приложенного электрического поля, $T$ - абсолютная температура, $N(W, T)$ - число электронов, падающих на единицу площади барьера за $1 \mathrm{~s}$ и имеющих энергию, близкую к $W ; D(W, E)$ - прозрачность потенциального барьера на контакте катода с внешней средой.

В модели Зоммерфельда число электронов, падающих на единицу площади за $1 \mathrm{~s}$, равно

$$
N(W, T)=\frac{4 \pi m k_{0} T}{h^{3}} \ln \left\{1+\exp \left(-\frac{W-W_{F}}{k_{0} T}\right)\right\},
$$

где $W_{F}$ - энергия Ферми металла, $k_{0}, h$ - постоянные Больцмана и Планка соответственно, $m$ - эффективная


Рис. 1. $a-$ контакт „металлический катод (1)-неметаллическая пленка (2) толщиной $d$-вакуум (3)“; $b$ - потенциальная энергия $W(x)$ электрона вблизи поверхности металла в области $x \geq 0$. Здесь: $W_{i e}(x)-$ классический электронный потенциал изображения; $W_{e}(x)-$ квантовый потенциал взаимодействия электрона с наведенной им быстрой поляризацией; $W(E)=-e E x-$ потенциальная энергия взаимодействия электрона с полем; $W_{a}$ - эффективная потенциальная энергия электрона внутри металла (постоянная величина); $\Phi=W_{F}-$ работа выхода (равная энергии Ферми); $W_{t m}$ - максимальное значение $W_{t}(x) ; W_{e}(0)=W_{t}(x=0)$.

масса электрона. Энергия отсчитывается от нуля для свободного электрона вне металла (рис. $1, b$ ), поэтому работа выхода $\Phi$ электрона равна по модулю энергии Ферми $W_{F}, W$ - энергия движения электрона в направлении, перпендикулярном к поверхности вне металла

$$
W=\frac{p^{2}(x)}{2 m}+W_{t}(x),
$$

где $p(x)$ - импульс электрона по нормали к поверхности, $W_{t}(x)$ - эффективная потенциальная энергия 
электрона (рис. 1, $b$ ), имеющая вид

$$
W_{t}(x)= \begin{cases}W_{e}(x)-e E x, & x \leq 0 \\ -W_{a}, & x<0\end{cases}
$$

где $W_{e}(x)$ - квантовая потенциальная энергия взаимодействия электрона с наведенной им быстрой поляризацией на контакте „металл-диэлектрик“(в пределе переходит в классический потенциал изображения при $x \gg R_{p}$, где

$$
R_{p}=\left(\hbar /\left(2 m \omega_{p}\right)^{(1 / 2)}\right.
$$

- радиус электронного полярона, $\omega_{p}$ - частота объемных плазменных колебаний).

В работе [18] и последующих работах в качестве потенциала $W_{e}(x)$ использован классический потенциал сил изображения, который имеет сингулярность при $x=0$, т. е. является некорректным как на самой границе контакта, так и вблизи нее $\left(x \sim R_{p}\right)$.

В работах [13-17] на основе поляронной теории потенциала и сил изображения получено выражение для квантового потенциала изображения $W_{e}(x)$, описывающего взаимодействие электрона с наведенной им быстрой поляризацией на контакте „металл-диэлектрик“, и справедливого во всей области значений $x \geq 0$ :

$$
\begin{aligned}
& W_{e}(x)=-e^{2} \int_{0}^{\infty} d \eta e^{-2 \eta x}\left[1-\frac{\eta^{2}}{2\left(\eta^{2}+k_{s}^{2}+k_{F_{s}}^{2}\right)}\right] \\
& \times \sum_{j=1,2} \frac{\varphi_{j}\left(\varepsilon_{j}(\eta)\right)}{\Omega_{j}^{2}(\eta)\left[1+R_{s_{j}}^{2} \eta^{2}\right]}-e^{2} \int_{0}^{\infty} k_{\perp} d k_{\perp} \int_{0}^{\infty} d k_{x} \\
& \times\left[1+e^{-2 k_{\perp} x}-2 e^{-k_{\perp} x} \cos \left(k_{x} x\right)\right] \\
& \times \frac{(\varepsilon-1)\left[1-\frac{k^{2}}{2\left(k^{2}+k_{S}^{2}+k_{V}^{2}\right)}\right]}{\varepsilon_{1} k^{2}\left(1+R_{V}^{2} k^{2}\right)},
\end{aligned}
$$

где

$$
\begin{aligned}
& \varphi_{1}\left(\varepsilon_{1}(\eta)\right)=\frac{1}{\left(\varepsilon_{1}(\eta)+1\right)^{2}}\left[\omega_{p V}\left(\frac{\left(\varepsilon_{1}(\eta)-1\right)}{\varepsilon_{1}(\eta)}\right)^{1 / 2}\right. \\
& \left.-\omega_{p} F_{12}\left(\Omega_{1}(\eta)\right)\right]^{2}\left[1+F_{12}^{2}\left(\Omega_{1}(\eta)\right)\right]^{-1}\left[1+R_{S_{1}}^{2}(\eta) \eta^{2}\right]^{-1}, \\
& \varphi_{2}\left(\varepsilon_{2}(\eta)\right)=\frac{1}{\left(\varepsilon_{2}(\eta)+1\right)^{2}}\left[-\omega_{p V}(\eta)\left(\frac{\left(\varepsilon_{2}(\eta)-1\right)}{\varepsilon_{2}(\eta)}\right)^{\frac{1}{2}}\right. \\
& \left.\times F_{21}\left(\Omega_{2}(\eta)\right)+\omega_{p}\right]^{2}\left[1+F_{21}^{2}\left(\Omega_{2}(\eta)\right)\right]^{-1}\left[1+F_{S_{2}}^{2}(\eta) \eta^{2}\right]^{-1} .
\end{aligned}
$$

Здесь $\varepsilon_{j}(\eta)$ - диэлектрическая функция квантового диэлектрика, для которой в [15] получено выражение

$$
\varepsilon_{j}(\eta)=1+\frac{\varepsilon-1}{\left[1+\frac{\eta^{2}}{\lambda^{2}}(\varepsilon-1)\right]\left(1+\frac{3 \eta^{2}}{4 \eta_{F}^{2}}\right)}
$$

$$
\lambda^{-1}=\frac{2(\varepsilon-1)}{\pi \sqrt{\varepsilon-1}} R_{p S}\left\{\sqrt{\varepsilon}+(\varepsilon-1) \operatorname{arctg} \sqrt{\varepsilon}-\frac{\pi}{2}(\varepsilon-1)\right\},
$$

где $R_{p S_{1,2}}=\left(\hbar /\left(2 m \Omega_{p S_{1,2}}\right)^{1 / 2}\right.$ - радиус поверхностного электронного полярона; $\Omega_{p S_{1,2}}$ - частоты поверхностных плазменных колебаний;

$$
k_{S}=2 \pi^{-1} k_{F} ; \quad k_{F}=\left(3 \pi^{2} N\right)^{1 / 3} .
$$

Явные выражения для величин $F_{12}\left(\Omega_{1}(\eta)\right) ; F_{21}\left(\Omega_{2}(\eta)\right)$, входящих в формулы (5) и (6), приведены в монографии [17].

Формулы (4)-(8) для квантового потенциала $W_{e}(x)$ являются громоздкими и не очень удобными для расчетов. В работах $[16,19,20]$ показано, что потенциальная энергия $W_{e}(x)$ может быть аппроксимирована с высокой точностью выражением

$$
\widetilde{W}_{e}(x) \approx-\frac{e^{2}}{\left(4 x+x_{0}\right) \varepsilon} .
$$

Здесь $x_{0}$ - параметр из теории поляронов, для которого получено выражение

$$
x_{0}=\frac{e^{2}}{\varepsilon W_{e}(0)},
$$

где

$$
\begin{aligned}
& W_{e}(0)=-e^{2} \int_{0}^{\infty} d \eta\left[1-\frac{\eta^{2}}{2\left(\eta^{2}+k_{s}^{2}+k_{F_{s}}^{2}\right)}\right] \\
& \times\left\{\frac{\varphi_{1}\left(\varepsilon_{1}(\eta)\right)}{\Omega_{1}^{2}(\eta)\left[1+R_{S_{1}}^{2} \eta^{2}\right]}+\frac{\varphi_{2}\left(\varepsilon_{2}(\eta)\right)}{\Omega_{2}^{2}(\eta)\left[1+R_{S_{2}}^{2} \eta^{2}\right]}\right\} .
\end{aligned}
$$

Представляет интерес оценить величину $x_{0}$ в сравнении с характерными масштабами теории. Для случая контакта „кристалл-вакуум“ без учета пространственной дисперсии интеграл (11) вычисляется точно, и для $x_{0}$ получаем выражение

$$
x_{0}=\frac{2 e^{2}\left(1-\frac{R_{S}^{2}}{R_{F}^{2}}\right)}{\pi \alpha_{p S} \hbar \Omega_{p S}\left(1+\frac{R_{S}^{2}}{R_{F}^{2}}+2 \frac{R_{S}^{2}}{R_{F}^{2}}\right)},
$$

где $R_{p S}, \alpha_{p S}$ - радиус поверхностного электронного полярона и константа электрон-плазмонного взаимодействия соответственно, $R_{S}=k_{S}^{-1}, R_{F}=k_{F}^{-1}$.

Учитывая, что

$$
\begin{gathered}
\alpha_{p S}=\frac{e^{2}}{\hbar}\left(1-\frac{1}{\varepsilon+1}\right)\left(\frac{m}{2 \hbar \Omega_{p} S}\right)^{1 / 2}, \\
R_{p S}=\left(\frac{m}{2 \hbar \Omega_{p S}}\right)^{1 / 2}
\end{gathered}
$$

получаем

$$
x_{0}=\frac{4\left(1-\frac{R_{S}^{2}}{R_{F}^{2}}\right)}{\left(1+\frac{R_{S}^{2}}{R_{F}^{2}}+2 \frac{R_{S}^{2}}{R_{F}^{2}}\right)} R_{p S},
$$


т. е. $x_{0} \sim R_{p S}$ и по порядку величины совпадает с радиусом электронного полярона. Для типичных значений параметров $m=(0.1-1) m_{0}$

$$
R_{p S} \sim(1-10) \cdot 10^{-8} \mathrm{~cm},
$$

т.е. составляет от одной до нескольких постоянных решетки.

Здесь необходимо подчеркнуть, что развиваемый в настоящей работе подход основан на теории электронных поляронов [10-12], согласно которой поляроны возникают при взаимодействии с плазмонами валентных электронов, характерная частота которых составляет величину порядка $10^{16} \mathrm{~s}^{-1}$. В связи с этим наведенная электроном поляризация безынерционно следует за движением электрона, превращая его в квазичастицу электронный полярон. В этом состоит основное отличие от моделей, учитывающих влияние электрон-фононного взаимодействия (частота $10^{13} \mathrm{~s}^{-1}$ ) на эмиссионные характеристики (см., например, [21]).

Отметим, что некоторыми авторами [22,23] параметр $x_{0}$ вводился как „обрезающий фактор“ в классическом потенциале изображения для устранения в нем „нефизической расходимости“ на поверхности кристалла $(x=0)$.

Для прозрачности барьера $D(E, W)$ на контакте можно записать

$$
D(E, W)=\left[1+\exp \left(-\frac{4 \pi i}{\hbar}\right) \int_{x_{1}}^{x_{2}} p(x) d x\right]^{-1},
$$

где

$$
p(x)=\sqrt{2 m\left(W-W_{t}(x)\right.}
$$

- зависящий от координаты импульс электрона, $W_{t}(x)$ - эффективная потенциальная энергия эмитированного электрона, которая с учетом выражений (3) и (9) может быть представлена в виде

$$
W_{t}=-\frac{e^{2}}{4 \varepsilon\left(x+x_{0} / 4\right)}-e E x .
$$

Тогда

$$
p(x)=\sqrt{2 m\left(W+\frac{e^{2}}{4 \varepsilon\left(x+\frac{x_{0}}{4}\right)}+e E x\right)} .
$$

(В выражениях (18)-(19) и далее использована система единиц CGSE.)

Пределы интегрирования в (16) находятся из условия $p(x)=0$. Тогда в соответствии с (19)

$$
W+\frac{e^{2}}{4 \varepsilon\left(x+x_{0} / 4\right)}+e E x=0 .
$$

Чтобы привести интеграл в (16), учитывающий (20), к виду, рассмотренному в [18], проведем замену

$$
z=x+x_{0} / 4
$$

Тогда из (20) следует

$$
W\left(1-\frac{e E x_{0}}{4 W}\right)+\frac{e^{2}}{4 \varepsilon z}+e E z=0 .
$$

Следуя [19], введем обозначение

$$
\gamma=1-\frac{e E x_{0}}{4 W} .
$$

Для нахождения пределов интегрирования из (20) получаем квадратное уравнение, решение которого имеет вид

$$
z_{1,2}=-\frac{\gamma W}{2 e E} \pm \sqrt{\frac{\gamma^{2} W^{2}}{4 e^{2} E^{2}}-\frac{e}{4 \varepsilon E}} .
$$

Вводим новую переменную

$$
y_{a}=\frac{e}{|\gamma W|} \sqrt{\frac{e E}{\varepsilon}} .
$$

Тогда, считая, что энергия $W$ принимает только отрицательные значения, можно записать

$$
z_{1,2}=-\frac{\gamma W}{2 e E}\left(1 \pm \sqrt{1-y_{a}^{2}}\right) .
$$

С учетом введенных обозначений интеграл в (16) приобретает вид

$$
I=-\frac{2 \sqrt{2 m}}{\hbar} i \int_{z_{1}}^{z_{2}}\left(\gamma W+\frac{e^{2}}{4 \varepsilon z}+e E z\right) d z
$$

Полученный интеграл аналогичен рассмотренному в [20]. Интегрирование в (27) приводит к появлению функции $v_{a}\left(y_{a}\right)$ :

$$
\begin{aligned}
v_{a}\left(y_{z}\right) & =\sqrt{\frac{1+\sqrt{1-y_{a}^{2}}}{2}}\left(E\left[\sqrt{\frac{2 \sqrt{1-y_{a}^{2}}}{1+\sqrt{1-y_{a}^{2}}}}\right]\right. \\
& \left.-\left(1-\sqrt{1-y_{a}^{2}}\right) K\left[\sqrt{\frac{2 \sqrt{1-y_{a}^{2}}}{1+\sqrt{1-y_{a}^{2}}}}\right]\right),
\end{aligned}
$$

где

$$
\begin{gathered}
K[k]=\int_{0}^{\pi / 2} \frac{d \theta}{\sqrt{1-k^{2} \sin ^{2} \theta}}, \\
E[k]=\int_{0}^{\pi / 2} \sqrt{1-k^{2} \sin ^{2} \theta} d \theta
\end{gathered}
$$

- эллиптические интегралы Эйлера 1-го и 2-го рода соответственно.

Отметим, что функция (28) отличается от функции Нордгейма [18] наличием в аргументе $y_{a}$ поляронного вклада.

Следуя [24], для случая $y_{a} \geq 1$ (что соответствует режиму автоэлектронной эмиссии) из (28) можно получить

$$
\left.v_{a}\left(y_{a}\right)=\sqrt{1+y_{a}}\right)\left(E\left[\sqrt{\frac{1-y_{a}}{1+y_{a}}}\right]-y_{a} K\left[\sqrt{\frac{1-y_{a}}{1+y_{a}}}\right]\right) .
$$


Аргумент $y_{a}$ может быть определен через аргумент у функции Нордгейма

$$
y_{a}=\frac{y}{\gamma \sqrt{\varepsilon}}
$$

Тогда из (28) для $v_{a}(y)$ (при условии $\varepsilon \approx 1$, которое выполняется для нанометрового диапазона толщины адсорбированной пленки) справедливо выражение

$$
\begin{aligned}
& v_{a}(y)=\sqrt{\frac{\gamma+\sqrt{\gamma^{2}-y^{2}}}{2 \gamma}}\left(E\left[\sqrt{\frac{2 \sqrt{\gamma^{2}-y^{2}}}{\gamma+\sqrt{\gamma^{2}-y^{2}}}}\right]\right. \\
& \left.-\frac{\gamma-\sqrt{\gamma^{2}-y^{2}}}{2 \gamma} K\left[\sqrt{\frac{2 \sqrt{\gamma^{2}-y^{2}}}{\gamma+\sqrt{\gamma^{2}-y^{2}}}}\right]\right) .
\end{aligned}
$$

Полный ток через контакт определяется выражением, аналогичным общей формуле (20) работы [18]:

$$
\begin{aligned}
j(E, T) & =\frac{k_{0} T}{2 \pi^{2}} \int_{-W_{a}}^{W_{t m}} \frac{\ln \left(1+\exp \left[-\frac{W-W_{F}}{k_{0} T}\right]\right)}{1+\exp \left[\frac{4}{3} \sqrt{2} E^{-\frac{1}{2}} y_{a}^{-\frac{3}{2}} v_{a}\left(y_{a}\right)\right]} d W \\
& +\frac{k_{0} T}{2 \pi^{2}} \int_{W_{t m}}^{\infty} \ln \left(1+\exp \left[-\frac{W-W_{F}}{k_{0} T}\right]\right) d W .
\end{aligned}
$$

Согласно [18], выражение (34) записано в единицах Хартри, т.е. $j$ выражено в единицах $m^{3} e^{9} \hbar^{-7}=2.37 \cdot 10^{14} \mathrm{~A} / \mathrm{cm}^{2} ; \quad E-$ в единицах $m^{2} e^{5} \hbar^{-4}=5.14 \cdot 10^{9} \mathrm{~V} / \mathrm{cm} ; W_{F}, k_{0} T, W, W_{a}, W_{t m}-$ в единицах $m e^{4} \hbar^{-2}=27.2 \mathrm{eV}$.

\section{Термоэлектронная эмиссия}

При высоких температурах эмиссионный ток обусловлен электронами, преодолевающими потенциальный барьер с энергией выше $W_{t m}$. В этом случае первым интегралом в (34) можно пренебречь. Тогда из (34) следует

$$
j_{R S}=\frac{4 \pi e m k_{0} T}{h^{3}} \int_{W_{t m}}^{\infty} \ln \left(1+\exp \left[-\frac{W-W_{F}}{k_{0} T}\right]\right) d W .
$$

Очевидно, что энергия эмитированных электронов в рассматриваемых условиях много больше энергии Ферми. Следовательно, подынтегральную функцию можно представить в виде

$$
\ln \left(1+\exp \left[-\frac{W-W_{F}}{k_{0} T}\right]\right) \approx \exp \left[-\frac{W-W_{F}}{k_{0} T}\right] .
$$

При этом из (35) с учетом (36) следует

$$
j_{R S}=\frac{4 \pi e m\left(k_{0} T\right)^{2}}{h^{3}} \exp \left(-\frac{W_{t m}-W_{F}}{k_{0} T}\right) .
$$
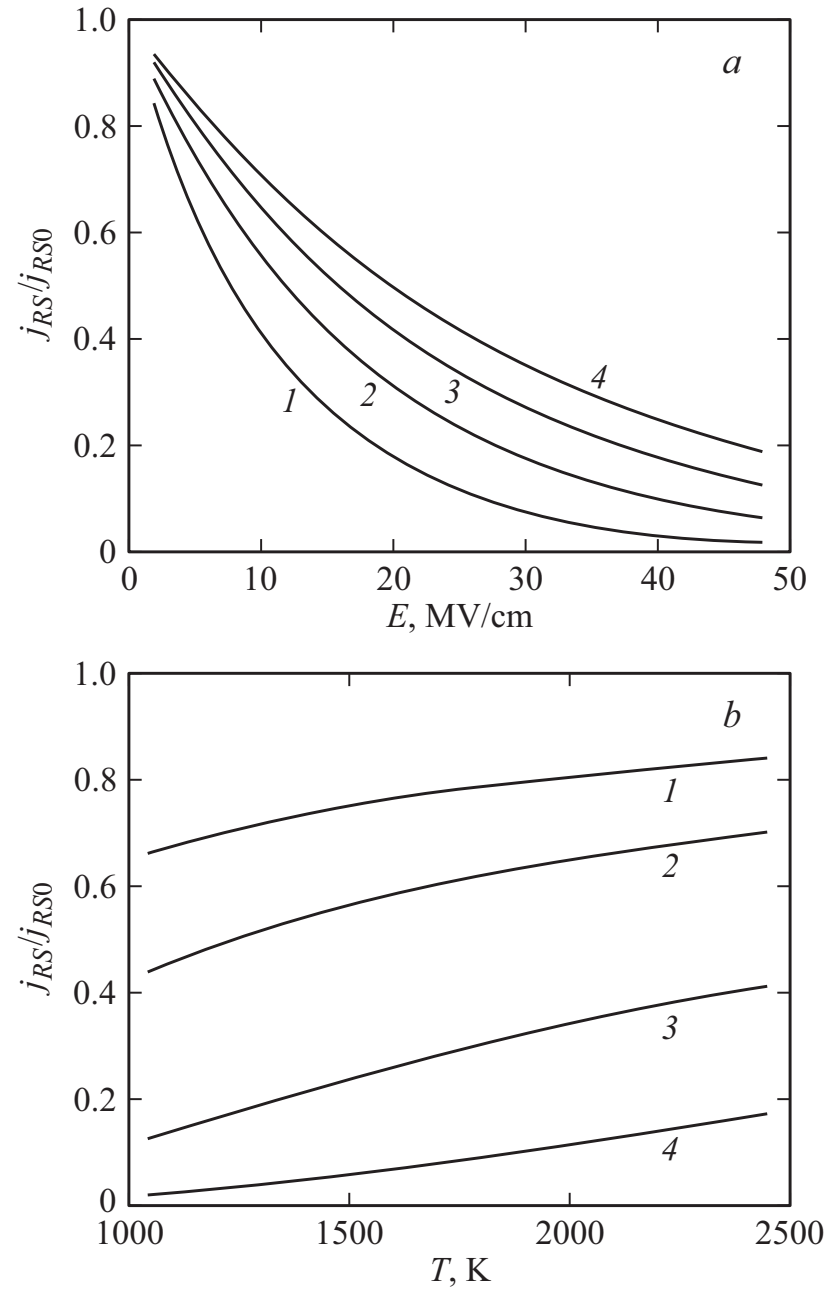

Рис. 2. $a-$ влияние квантовых сил изображения на величину плотности эмиссионного тока: полевая зависимость для различных значений температуры: $1-1000,2-1500,3-$ $2000,4-2500 \mathrm{~K} ; b-$ влияние квантовых сил изображения на величину плотности эмиссионного тока: температурная зависимость для различных значений напряженности электрического поля: $1-5,2-10,3-25,4-50 \mathrm{MV} / \mathrm{cm}$.

Определим энергию $W_{t m}$. Потенциальная энергия электрона вне металлического катода определяется выражением

$$
W_{t}=W_{F}+\Phi-\frac{e^{2}}{\varepsilon\left(4 x+x_{0}\right)}-e E x .
$$

Здесь $\Phi$ - работа выхода материала катода.

Энергия $W_{t}$ достигает максимума в точке

$$
x_{m}=\frac{1}{2} \sqrt{\frac{e}{E}}-\frac{x_{0}}{4} .
$$

Подставляя выражение $(39)$ в (38), для $W_{m}$ получаем

$$
W_{t m}=W_{F}+\Phi-\frac{1}{\varepsilon} \sqrt{e^{3} E}+\frac{e E x_{0}}{4 \varepsilon} .
$$

Из формул (37) и (40) следует окончательное выражение для плотности тока поляронной термоэлектронной 


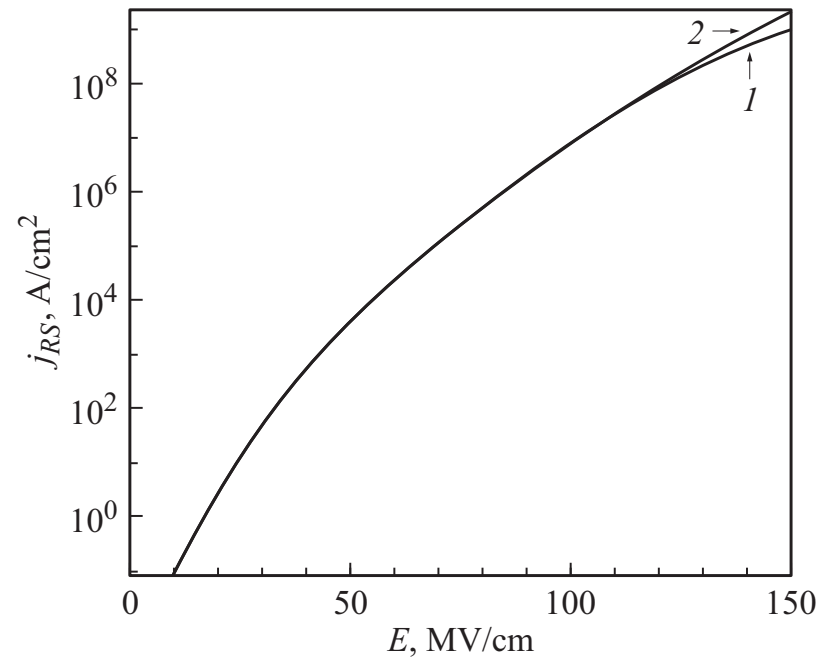

Pис. 3. Зависимость тока термоэлектронной эмиссии от напряженности электрического поля. Кривая 1 рассчитана по точной формуле (35), кривая 2 - по обобщенной формуле Ричардсона-Шоттки (41). Значения параметров: $\varepsilon=1$, $\Phi=4 \mathrm{eV}, m=m_{0}, T=1500 \mathrm{~K}$.

эмиссии, представляющее собой обобщенную формулу Ричардсона-Шоттки:

$$
j_{R S}=\frac{4 \pi e m k_{0}^{2}}{h^{3}} T^{2} \exp \left[\frac{1}{k_{0} T}\left(-\Phi+\frac{1}{\varepsilon} \sqrt{e^{3} E}-\frac{e E x_{0}}{4 \varepsilon}\right)\right] .
$$

Как показано в [18], формула Ричардсона-Шоттки (а значит, и формула (41)) справедлива вплоть до полей напряженностью порядка $50 \mathrm{MV} / \mathrm{cm}$.

Оценим влияние поляронного эффекта (т. е. квантового характера сил изображения) на величину эмиссионного тока Ричардсона-Шоттки. Без его учета плотность тока определяется вытекающим из (41) выражением:

$$
j_{R S_{0}}=\frac{4 \pi e m k_{0}^{2}}{h^{3}} T^{2} \exp \left[\frac{1}{k_{0} T}\left(-\Phi+\frac{1}{\varepsilon} \sqrt{e^{3} E}\right)\right] .
$$

Тогда отношение $j_{R S} / j_{R S 0}$ характеризует влияние поляронного характера туннелирования на величину эмиссионного тока:

$$
\frac{j_{R S}}{j_{R S 0}}=\exp \left(-\frac{e E x_{0}}{4 \pi k_{0} T}\right) .
$$

Результаты расчетов для некоторых усредненных значений $\varepsilon=3, x_{0}=0.3 \mathrm{~nm}, \Phi=4.5 \mathrm{eV}$ приведены на рис. 2. Графики на рис. 2 свидетельствуют о большом влиянии электронного поляронного эффекта на эмиссионные характеристики катодов в сильных электрических полях. Причем в области значений напряженности полей $E \geq 10 \mathrm{MV} / \mathrm{cm}$ это влияние растет с ростом поля (достигая примерно двух порядков при напряженности электрического поля $50 \mathrm{MV} / \mathrm{cm})$ и снижается с ростом температуры.
На рис. 3 приведены зависимости плотности тока Ричардсона-Шоттки, рассчитанные по точной формуле (35) и приближенной формуле (41). Эти зависимости практически совпадают во всей области, где справедливо уравнение Ричардсона-Шоттки.

Уменьшение эмиссионного тока с ростом напряженности электрического поля связано с увеличением эффективной работы выхода Ф. Как следует из (41) и (42), в расчетах необходимо использовать значение

$$
\bar{\Phi}=\Phi+\frac{e E x_{0}}{4 \varepsilon}
$$

Таким образом, показано, что учет электронного поляронного эффекта приводит к увеличению работы выхода во всем интервале полей и температур. В области напряженности поля $E>5 \mathrm{MV} / \mathrm{cm}$ это приводит к снижению плотности эмиссионного тока более чем на порядок, что связано с дополнительной работой поля по перемещению как самого электрона, так и следующего за ним поляризационного облака при туннелировании электронного полярона через барьер. Формула (44) для эффективной работы выхода $\bar{\Phi}$ подтверждает результаты работ $[25,26]$, в которых экспериментально измеренная величина контактного барьера в процессе внутреннего фотоэффекта в структуре $\mathrm{Al}-\mathrm{SiO}_{2}$ - вакуум оказалась больше, чем действительная высота барьера, примерно на $0.2 \mathrm{eV}$.

\section{Автоэлектронная эмиссия}

Интегрирование выражения (34) с учетом (27) и (31) приводит к уравнению, аналогичному уравнению Мерфи-Гуда [18]:

$$
\begin{aligned}
& j\left(E, T, x_{0}\right)=\frac{e^{3} E^{2}}{8 \pi h \varepsilon^{2} \Phi t_{a}^{2}\left(y_{a}\right)} \cdot \frac{\pi c_{a} k_{0} T}{\sin \left(\pi c_{a} k_{0} T\right)} \\
& \times \exp \left[-\frac{8 \pi \varepsilon \sqrt{2 m} \Phi^{3 / 2}}{3 e h E} v_{a}\left(y_{a}\right)\right] .
\end{aligned}
$$

Отметим, что кроме функции $v_{a}\left(y_{a}\right)$ в формуле (45) появляются новые функции $t_{a}\left(y_{a}\right)$ и $c_{a}\left(y_{a}\right)$ (содержащие поляронные вклады), которые можно выразить через аргумент у функции Нордгейма:

$$
\begin{gathered}
t_{a}\left(y_{a}\right)=\bar{\gamma} \sqrt{\frac{y_{a}}{2}}\left\{2 E\left[\sqrt{\frac{y_{a}-y}{2 y_{a}}}\right]-K\left[\sqrt{\frac{y_{a}-y}{2 y_{a}}}\right]\right\}, \\
c_{a}\left(y_{a}\right)=\frac{4 \pi \varepsilon \sqrt{2 m \Phi}}{e h E} t_{a}\left(y_{a}\right)
\end{gathered}
$$

где

$$
\bar{\gamma}=1+\frac{e E x_{0}}{4 \Phi}
$$

Температурная зависимость тока автоэлектронной эмиссии определяется вторым предэкспоненциальным сомножителем уравнения (45):

$$
\alpha(T, E)=\frac{\pi c_{a} k_{0} T}{\sin \left(\pi c_{a} k_{0} T\right)} .
$$




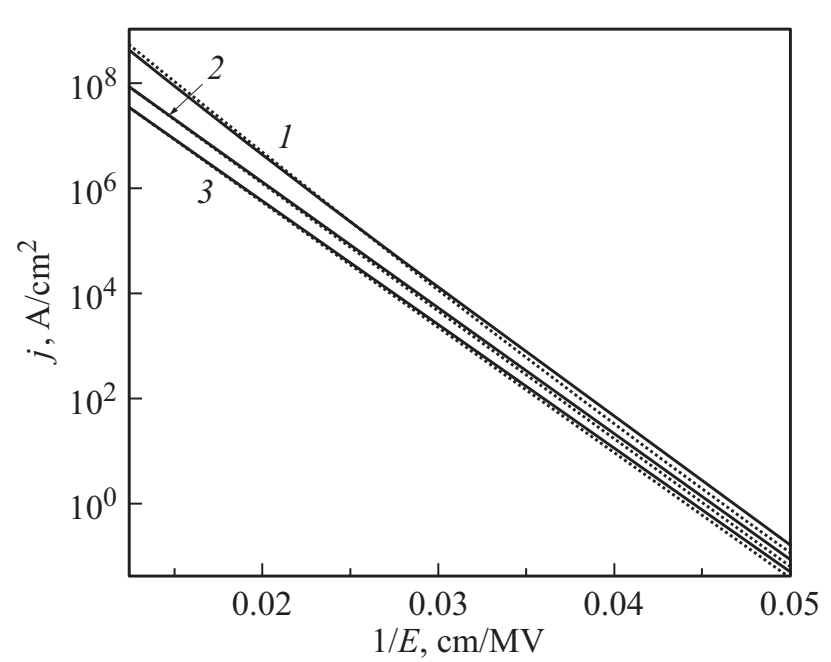

Рис. 4. Зависимости плотности тока автоэлектронной эмиссии, рассчитанные на основе формулы (45) (сплошные линии) и по экстраполяционной формуле (52) (пунктирные линии) для различных значений параметра $x_{0}(1-0,2-0.5,3-$ $1.0 \mathrm{~nm}$ ), от напряженности $E$ электрического поля в области сильных полей $(E=(30-80) \mathrm{MV} / \mathrm{cm})$. Значения других параметров теории: $\varepsilon=1, \Phi=4 \mathrm{eV}, m=m_{0}, T=500 \mathrm{~K}$.

Если $\pi c_{a} k T$ настолько мало, что $\alpha(T, E) \simeq 1$, а поляронный эффект пренебрежимо мал (т.е. $x_{0}=0$ ), то формула (45) приходит в формулу Фаулера-Нордгейма:

$$
j_{F-N}(E)=\frac{e^{3} E^{2}}{8 \pi h e^{2} \Phi t^{2}(y)} \exp \left[-\frac{8 \pi \varepsilon \sqrt{2 m} \Phi^{3 / 2}}{3 e h E} v(y)\right] .
$$

Следует отметить, что формулы (45) и (50) имеют ограничения по значениям входящих в эти соотношения физических параметров - температуры, работы выхода и напряженности электрического поля.

Таким образом, формулу (45) можно считать обобщенным выражением плотности тока термоавтоэлектронной эмиссии на контакте „металл-диэлектрик“, покрытого адсорбированной неметаллической пленкой, учитывающей вклад поляронного эффекта и его возрастающую роль с увеличением напряженности электрического поля и температуры. Она отличается от классической формулы для плотности эмиссионного тока, приведенной в [18], наличием в аргументе функций $v_{a}\left(y_{a}\right)$ и $t_{a}\left(y_{a}\right)$,поляронного вклада“".

Формула (45) в пределе низких температур $(T \rightarrow 0)$, при которых $\alpha(T, E)=1$, и высоких полей может быть преобразована к более простому виду, если использовать аппроксимацию для функций $v_{a}\left(y_{a}\right)$ и $t_{a}^{2}\left(y_{a}\right)$, найденную в работе [27] и примененную авторами [28] для определения площади барьера полевой эмиссии:

$$
v_{a}\left(y_{a}\right) \approx 0.95-1.03 y_{a}^{2}, \quad t_{a}^{2}\left(y_{a}\right) \approx 1.1 .
$$

Подставляя (50) в (45), с учетом (32), (25) получаем выражение для плотности тока автоэлектронной эмиссии:

$$
j_{a p p r}(E)=\frac{e^{3} E^{2}}{8.8 \pi h \varepsilon^{2} \Phi} \exp \left[-\frac{8 \pi \sqrt{2 m} \Phi^{3 / 2}}{3 e h E_{\mathrm{eff}}}\right],
$$

где $E_{\text {eff }}$ имеет вид

$$
E_{e f f}=\frac{E}{0.95-\frac{1.03 e^{3} E}{\varepsilon\left(\Phi+\frac{E x_{0}}{4}\right)^{2}}}
$$

и включает в себя поляронный параметр $x_{0}$.

На рис. 4 представлена полевая зависимость плотности тока автоэлектронной эмиссии, рассчитанного соответственно по формуле (45) (сплошные линии) и по экстраполяционной формуле (52) (пунктирные линии) для различных значений параметра $x_{0}$ $\left(x_{0}=0 \mathrm{~nm}, x_{0}=0.5 \mathrm{~nm}, x_{0}=1.0 \mathrm{~nm}\right)$, от напряженности электрического поля в области сильных полей $(E=30-80 \mathrm{MV} / \mathrm{cm})$.

Приведенные на рис. 4 зависимости плотности тока Фаулера-Нордгейма в указанном диапазоне полей различаются менее чем на $3-5 \%$, в то же время имеет место сильная зависимость от параметра $x_{0}$, описывающего поляронный эффект.

Отметим, что во всей области значений полей на рис. 4 при $x_{0}=0$ имеет место совпадение с результатами известной теории Мэрфи-Гуда [18], в которой термои автоэлектронная эмиссия рассматриваются с единой точки зрения. Результаты этой теории подтверждаются многочисленными экспериментами.

Как и в случае термоэлектронной эмиссии, уменьшение плотности тока автоэлектронной эмиссии с ростом $x_{0}$ объясняется увеличением эффективной работы выхода электрона в сильном электрическом поле, обусловленным плазмонным поляронным эффектом.

Следует отметить, что экстраполяционные формулы (41) - для плотности тока термоэлектронной эмиссии и (52) - для плотности тока автоэлектронной эмиссии, как следует из рис. 3 и 4 , дают результаты, практически совпадающие с результатами, полученными по точным формулам (35) и (45) соответственно, в интервале полей от 10 до $100 \mathrm{MV} / \mathrm{cm}$.

\section{Заключение}

Применение поляронной теории к рассмотрению физических процессов на контакте металл-диэлектрик или металл-адсорбированная диэлектрическая нанопленка позволяет устранить сингулярность потенциальной энергии туннелирующего электрона на эмитирующей границе (при $x=0)$. При полях $E>5 \cdot 10^{6} \mathrm{~V} / \mathrm{cm}$, когда ширина потенциального барьера соизмерима с радиусом электронного полярона, квантовый вклад в плотность эмиссионного тока становится существенным. Этот вклад определяется параметром $x_{0}$, который связан с радиусом туннелирующего полярона. При этом с ростом 
напряженности электрического поля влияние поляронного эффекта возрастает, что обусловлено увеличением эффективной работы выхода электрона. Этот эффект частично ослабляется при учете реального значения диэлектрической проницаемости материала адсорбированной нанопленки.

В то же время в рамках данной модели не находят объяснения высокие значения коэффициента усиления поля, определяемые из характеристик Фаулера-Нордгейма. Возможная причина этого связана с неучетом влияния проникновения внешнего электрического поля на поляронную электронную эмиссию. Этот анализ предполагается сделать в дальнейших работах.

\section{Финансирование работы}

Работа поддержана грантом РФФИ № 17-08-01282.

\section{Конфликт интересов}

Авторы заявляют, что у них нет конфликта интересов.

\section{Список литературы}

[1] Mesyats G.A., Proskurovsky D.I. Pulsed Electrical Discharge in Vacuum. Berlin: Springer, 1989. 293 p.

[2] High voltage vacuum insulation: Basic concepts and technological practice / Ed. by R.V. Latham. London: Academic Press, 1995. 594 p.

[3] Cox B.M., Williams W.T. // J. Phys. D: Appl. Phys. 1977. Vol. 10. N 3. L5-L10.

[4] Месяи Г.А. Эктоны в вакуумном разряде: пробой, искра, дуга. М.: Наука, 2000. [Mesyats G.A. Cathode Phenomena in a Vacuum Discharge: The Breakdown, the Spark, and the Arc. M.: Nauka, 2000.]

[5] Anders A. Cathodic Arcs: From Fractal Spots to Energetic Condensation. NY.: Springer, 2008. 540 p.

[6] A $3 \mathrm{TeV} e^{+} e^{-}$Linear Collider Based on CLIC Technology : the CLIC study team / Ed. by G. Guignard. Geneva, 2000. 76 p. (CERN Report; 2000-008).

[7] Wuensch W. Tech. Rep. // CERN-OPEN-2014-028. CLICNote-1025. CERN, Geneva, May 2013.

[8] Barengolts S.A., Mesyats V.G., Oreshkin V.I., Oreshkin E.V., Khishchenko K.V., Uimanov I.V., Tsventoukh M.M. // Phys. Rev. Accelerat. Beams. 2018. Vol. 21. N 6. P. 061004.

[9] Korolev Yu.D., Mesyats G.A. Physics of Pulsed Breakdown in gases. Yekaterinburg: URO-press, 1998. $274 \mathrm{p}$.

[10] Toyozawa Y. // Progr. Theor. Phys. 1954. Vol. 12. N 3. P. $421-436$

[11] Hermanson J. // Phys. Rev. 1972. Vol. 6. N 6. P. 2427-2432.

[12] Hermanson J., in: Elementary Excitations in Solids, Molecules, and Atom. Part B. Springer, 1974. P. 199-211.

[13] Берил С.И., Покатилов Е.П. // ФТП. 1978. Т. 2. C. 2030-2033. [Beril S.I., Pokatilov E.P. // Semiconductors. 1978. Vol. 12. P. $1207-1208$.

[14] Покатилов Е.П., Берил С.И., Фомин В.М. // Поверхность (Физика, химия, механика). 1988. Т. 5. С. 5-12.

[15] Pokatilov E.P., Beril S.I., Fomin V.M. // Phys. Stat. Sol. B. 1988. Vol. 147. P. 163-172.
[16] Beril S.I., Pokatilov E.P., Goryachkovskii E.P., Semenovskaya N.N. // Phys. Stat. Sol. B. 1993. Vol. 176. P. $347-353$.

[17] Покатилов Е.П., Берил С.И., Фомин В.М. Колебательные возбуждения, поляроны и экситоны в многослойных системах и сверхрешетках. Кишинев: Штиинца, 1990. C. $155-156$.

[18] Murphy E.I., Good A.H. // Phys. Rev. 1956. Vol. 162. P. 1464-1473.

[19] Barengolts Y.A., Beril S.I. // IEEE Trans. Plasma Sci. 2014. Vol. 42. P. 3109-3112.

[20] Barengolts Y.A., Beril S.I. // Proc. $3^{\text {rd }}$ Int. Conf. on Nanotechnologies and Biomedical Engineering. 2016. Vol. 55. P. 230-233.

[21] Reich K.V., Eidelman E.D. // Euro Phys. Lett. 2009. Vol. 85. P. 47007.

[22] Эдельман В.С. // УФН. 1980. Т. 130. Вып. 4. С. 675-704. [Edel'man V.S. // Soviet Phys. Uspekhi. 1980. Vol. 23. N 4. P. 227-244. DOI: 10.1070/PU1980v023n04ABEH004711

[23] Шикин В.Б., Монарха Ю.П. // ФНТ. 1975. Т. 1. № 8. C. 957-983.

[24] Modinos A. Field, Thermionic, and Secondary Electron Emission Spectroscopy. NY.: Plenum Press, 1984. 320 p.

[25] Harstein A., Weinberg Z.A. // Phys. Rev. 1979. Vol. 20. N 4. P. $1335-1338$.

[26] Harstein A., Weinberg Z.A. // J. Phys. C. Solid State Phys. 1978. Vol. 11. N 11. L469-L473.

[27] Шредник B.Н. В кн.: Ненакаливаемые катоды. М.: Сов. радио, 1974. С. 166-169.

[28] Попов Е.О., Колосько А.Г., Чумак М.А., Филиппов С.В. // ЖТФ. 2019. Т. 89. Вып. 10. С. 1615-1625. 